Inferring the flavor of high-energy astrophysical neutrinos at their sources

Bustamante, Mauricio; Ahlers, Markus

Published in:

Physical Review Letters

DOI:

10.1103/PhysRevLett.122.241101

Publication date:

2019

Document version

Publisher's PDF, also known as Version of record

Citation for published version (APA):

Bustamante, M., \& Ahlers, M. (2019). Inferring the flavor of high-energy astrophysical neutrinos at their sources.

Physical Review Letters, 122, [241101]. https://doi.org/10.1103/PhysRevLett.122.241101 


\title{
Inferring the Flavor of High-Energy Astrophysical Neutrinos at Their Sources
}

\author{
Mauricio Bustamante ${ }^{1,2, *}$ and Markus Ahlers ${ }^{1, \dagger}$ \\ ${ }^{1}$ Niels Bohr International Academy and Discovery Centre, Niels Bohr Institute, University of Copenhagen, \\ DK-2100 Copenhagen, Denmark \\ ${ }^{2}$ DARK, Niels Bohr Institute, University of Copenhagen, DK-2100 Copenhagen, Denmark
}

(Received 4 February 2019; revised manuscript received 8 April 2019; published 20 June 2019)

\begin{abstract}
The sources and production mechanisms of high-energy astrophysical neutrinos are largely unknown. A promising opportunity for progress lies in the study of neutrino flavor composition, i.e., the proportion of each flavor in the flux of neutrinos, which reflects the physical conditions at the sources. To seize it, we introduce a Bayesian method that infers the flavor composition at the neutrino sources based on the flavor composition measured at Earth. We find that the present data from the IceCube neutrino telescope favor neutrino production via the decay of high-energy pions and rule out production via the decay of neutrons. In the future, improved measurements of flavor composition and mixing parameters may single out the production mechanism with high significance.
\end{abstract}

DOI: 10.1103/PhysRevLett.122.241101

Introduction.-High-energy astrophysical neutrinos with $\mathrm{TeV}-\mathrm{PeV}$ energies provide crucial and unique information to understand the nonthermal Universe [1,2]. Yet, though firmly detected [3-7], they have a largely unknown origin. They likely come predominantly from extragalactic sources [2,8-11], but, to date, no pointlike source is known with certainty, notwithstanding noteworthy recent findings [12,13]. In the future, improved event statistics, reduced systematic uncertainties, and combined multimessenger analyses will boost the prospects of discovering sources $[14,15]$.

A complementary opportunity for progress, accessible already today, lies in measuring the flavor composition of high-energy astrophysical neutrinos, i.e., the relative number of neutrinos of each flavor. The flavor composition that neutrinos are emitted with is the result of production processes that depend on the physical conditions in the astrophysical sources. Therefore, flavor measurements can help to discriminate between candidate source classes [16-20]. After emission, as neutrinos propagate en route to Earth, flavor oscillations modify the composition. Neutrino telescopes, like IceCube, measure the flavor composition of the arriving flux. Based on it, one can, in principle, infer the composition at the sources.

Yet, existing analyses are either largely focused on inferring the flavor composition at Earth from data [21-27] or confined to assessing the compatibility of the flavor composition measured at Earth with expectations from a few idealized scenarios of neutrino production. We follow an alternative strategy, hitherto unexplored, that provides more insight. Using Bayesian statistics, we infer the composition at the sources based on the composition measured at neutrino telescopes, accounting for the uncertainties in its measurement and in the neutrino mixing parameters that drive oscillations.

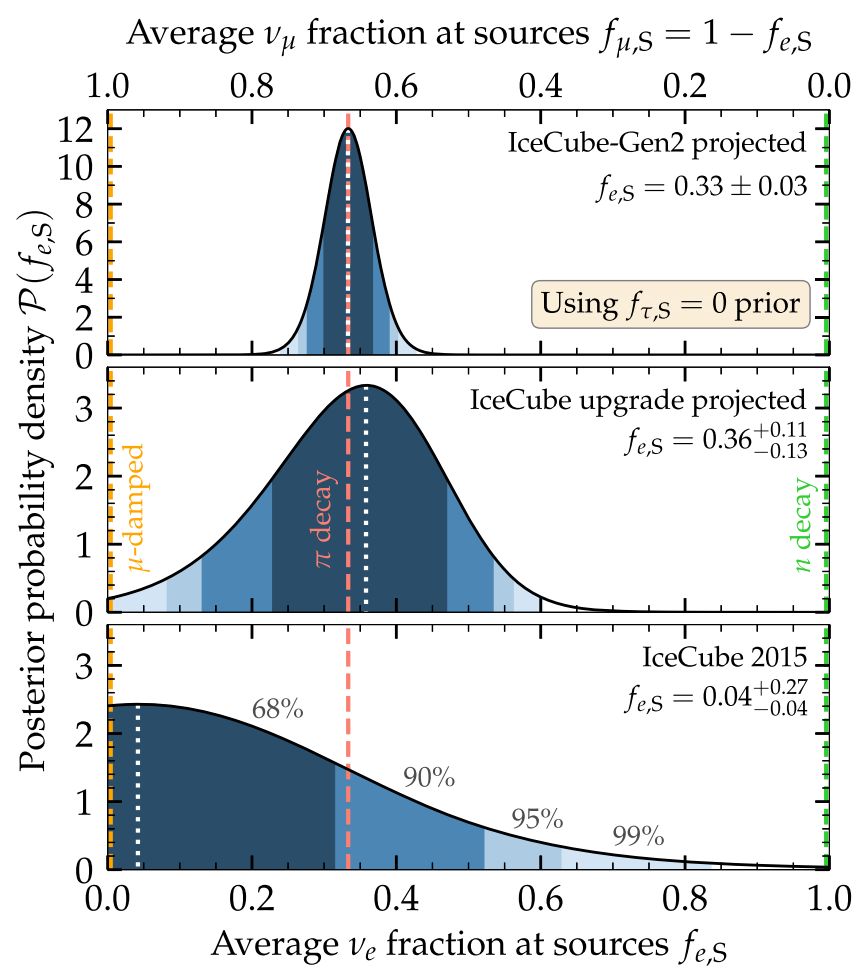

FIG. 1. Flavor composition of high-energy astrophysical neutrinos at their sources, inferred from present IceCube measurements [23] (bottom) and from the projected sensitivities of the near-future IceCube upgrade [28] (center) and planned IceCubeGen2 [29] (top), assuming production by pion decay. Here we enforce a prior of no $\nu_{\tau}$ production, i.e., $f_{\tau, S}=0$. We show the most probable values (white dotted lines) and credible intervals (blue shaded regions) of $f_{e, S}$ and mark physically motivated neutrino production scenarios: pion decay, muon-damped, and neutron decay. 
Figure 1 shows our results obtained using published and projected flavor measurements in IceCube. We report results in terms of flavor ratios $f_{\alpha, S}(\alpha=e, \mu, \tau)$, i.e., the relative contribution of $\nu_{\alpha}+\bar{\nu}_{\alpha}$ to the total emission. We find that the present data favor neutrino production via the decay of high-energy pions and the synchrotron cooling of intermediate muons in strong magnetic fields and strongly disfavor production via neutron decay.

Producing astrophysical neutrinos.-We expect astrophysical sources of high-energy neutrinos to accelerate protons beyond $\mathrm{PeV}$ energies via collisionless shocks in magnetized environments. High-energy protons interact with ambient matter [30-32] and photons [31,33,34] to produce high-energy pions. When they decay, they produce $\mathrm{TeV}-\mathrm{PeV}$ neutrinos via $\pi^{+} \rightarrow \mu^{+}+\nu_{\mu}$, followed by $\mu^{+} \rightarrow \bar{\nu}_{\mu}+\nu_{e}+e^{+}$, and their charge-conjugated processes. Thus, neutrinos are nominally expected to be produced with flavor ratios $\left(N_{e}: N_{\mu}: N_{\tau}\right)=(1: 2: 0)_{S}$, with $N_{\alpha}$ the sum of $\nu_{\alpha}$ and $\bar{\nu}_{\alpha}$. Because at these energies it is difficult to disentangle the relative contribution of $\nu$ and $\bar{\nu}$ in neutrino telescopes, existing analyses typically assume that they contribute equally to the flux. Thus, below, $\nu_{\alpha}$ refers to $\nu_{\alpha}+\bar{\nu}_{\alpha}$, unless otherwise indicated. Interaction with matter in the sources likely does not modify the flavor ratios after production [35-39].

Other production mechanisms may affect the flavor composition; we highlight two possibilities. First, if the muons from pion decay significantly lose energy before decaying, e.g., by synchrotron radiation in a strong magnetic field, neutrinos born from muon decay have lower energies. In this "muon-damped" scenario, the high-energy flavor ratios are $(0: 1: 0)_{S}$. Second, neutrons coproduced with pions beta decay into $\bar{\nu}_{e}$, yielding $(1: 0: 0)_{S}$. Yet, these neutrinos are $\sim 100$ times less energetic than those made in pion decays. Throughout, we use the three physically motivated scenarios-full pion decay, muon damping, and neutron decay-as benchmarks.

Production of $\nu_{\tau}$ is expected to be strongly suppressed, since it would require producing rare mesons, like $D_{s}^{ \pm}$. Below, we explore the full breadth of production mechanisms-including those with large $\nu_{\tau}$ content-and the scenario that enforces no $\nu_{\tau}$ production.

The flavor ratios might evolve with energy $[34,40-43]$. In the main text, we assume that they are measured in a single energy bin, so that any flavor evolution is hidden. This is the current experimental status [22,23]. However, future neutrino analyses will allow us to study the flavor composition in multiple high-energy bins; see Supplemental Material [44] for the case of IceCube-Gen2.

Neutrino oscillations.-Because a neutrino of a given flavor $\nu_{\alpha}$ is a superposition of neutrino mass eigenstates $\nu_{i}$ $(i=1,2,3)$, it can change flavor as it propagates. The connection between the flavor and mass bases is represented by the Pontecorvo-Maki-Nakagawa-Sakata (PMNS) unitary mixing matrix $\mathbf{U}$. Following convention, we parametrize it in terms of three mixing angles, $\theta_{12}, \theta_{23}$, and $\theta_{13}$, and one $C P$-violation phase, $\delta_{C P}$, whose values are measured in numerous oscillation experiments.

For $\mathrm{TeV}-\mathrm{PeV}$ astrophysical neutrinos, the probability $P_{\alpha \beta}$ of the flavor transition $\nu_{\alpha} \rightarrow \nu_{\beta}$ oscillates rapidly. Because of the energy spread of neutrinos and the limited energy resolution of detectors [45], flavor oscillations average out and the probability is [46] $P_{\alpha \beta}=$ $\sum_{i=1}^{3}\left|U_{\alpha i}\right|^{2}\left|U_{\beta i}\right|^{2}$, where $U_{\alpha i}$ are elements of the PMNS matrix. Thus, the flavor ratios at Earth are $f_{\alpha, \oplus}=$ $\sum_{\beta=e, \mu, \tau} P_{\beta \alpha} f_{\beta, S}$. If neutrinos are produced in the full pion decay chain and the probability is evaluated at the best-fit values of the mixing parameters, the flavor ratios at Earth are close to $(1: 1: 1)_{\oplus}$; this is the nominal expectation. Flavor ratios can be used to probe fundamental neutrino physics, though we do not explore this possibility here; see, e.g., Refs. [16,43,47-57].

Mixing parameters.-Presently, $\theta_{12}$ and $\theta_{13}$ are known at $1 \sigma$ to within $2 \%, \theta_{23}$ to within $8 \%$, and $\delta_{C P}$ to within $20 \%$. This translates into uncertainties of around $20 \%$ in transition probabilities, which we account for below. For our analysis, we adopt the allowed ranges of mixing parameters from the NuFit 3.2 global fit to oscillation data $[58,59]$, assuming normal neutrino mass ordering $\left(s_{i j} \equiv \sin \theta_{i j}\right)$ : $s_{12}^{2}=0.307_{-0.012}^{+0.013}, s_{23}^{2}=0.538_{-0.069}^{+0.033}, s_{13}^{2}=(2.206 \pm 0.075) \times$ $10^{-2}$, and $\delta_{C P}=\left(234_{-31}^{+43}\right)^{\circ}$. The phase $\delta_{C P}$ has only a marginal influence on the flavor composition at Earth, since it appears in the flavor-transition probabilities suppressed by two or four powers of the small angle $s_{13}$. Using inverted ordering does not affect our conclusions; we show this in Supplemental Material [44].

We account for the uncertainties in the mixing parameters via their probability density functions $(\mathrm{PDFs}) \mathcal{P}$. For each parameter in the set $\boldsymbol{\theta} \equiv\left(s_{12}, s_{23}, s_{13}, \delta_{C P}\right)$, we approximate its PDF as a normal distribution with the mean and standard deviation computed, respectively, from the best-fit and largest $1 \sigma$ error above. This is justified, because the $\Delta \chi^{2}$ curves that represent their uncertainties are nearly symmetric around the best-fit values $[58,59]$. Future implementations of our proposed method could use refined PDFs built directly from the $\Delta \chi^{2}$ curves.

Figure 2, left, shows for the three benchmark production scenarios that varying the mixing parameters within their $3 \sigma$ uncertainties results in small allowed regions of flavor ratios at Earth. Yet, these uncertainties, small though they seem, are an important limiting factor when reconstructing flavor ratios at the sources.

Measuring flavor at Earth.-IceCube is presently the largest, most sensitive detector of high-energy neutrinos [2]. It instruments a gigaton of clear Antarctic ice with an array of strings of photomultipliers [60]. When a highenergy neutrino interacts with a nucleon in the vicinity of the detector, final-state charged particles initiate particle showers that radiate Cherenkov light, which is collected by the photomultipliers. In the $\mathrm{TeV}-\mathrm{PeV}$ neutrino energy range, IceCube detects two types of neutrino-induced event 

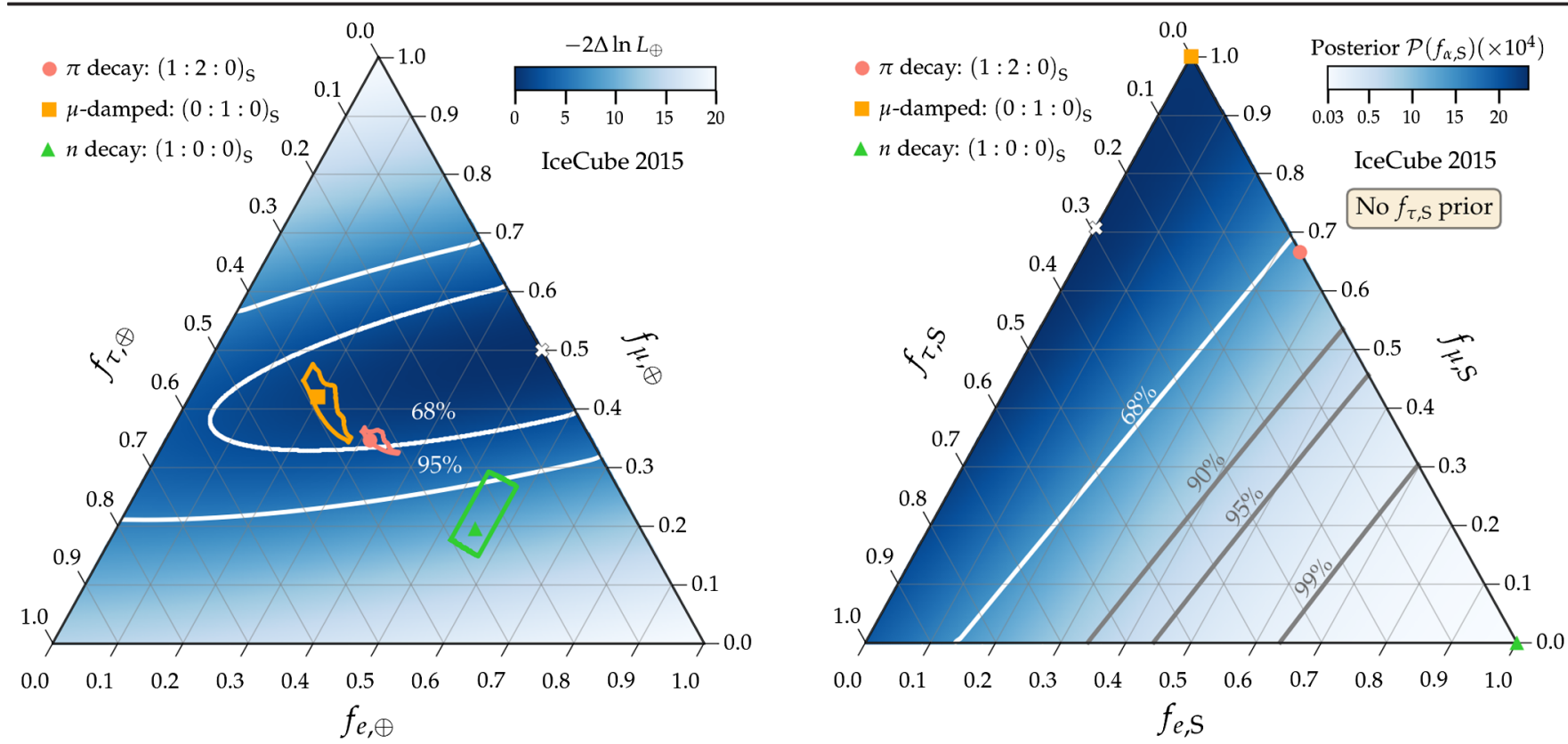

FIG. 2. Left: Flavor composition of high-energy astrophysical neutrinos at Earth, approximating current IceCube measurements [23], expressed in terms of variations in the likelihood, $-2 \Delta \ln \mathcal{L}_{\oplus}$. The contours show the $68 \%$ and $95 \%$ confidence regions; This triangle was produced by the IceCube Collaboration using a frequentist approach. We include expectations from three benchmark production scenarios, computed with mixing parameters fixed at their best-fit values—shown as symbols-and varied within their $3 \sigma$ ranges [58,59] — shown as bounded regions. Right: Flavor composition at the neutrino sources, inferred based on current measurements of flavor in IceCube and of mixing parameters in oscillation experiments [58,59]. We assume no prior on the flavor composition at the sources. The contours show the $68 \%, 90 \%, 95 \%$, and $99 \%$ credible regions; this triangle was produced by the procedure introduced here using a Bayesian approach.

topologies: elongated tracks from high-energy muonsinitiated mainly by interactions of $\nu_{\mu}$-and approximately spherical showers from electromagnetic and hadronic cascades-initiated by all flavors but predominantly by $\nu_{e}$ and $\nu_{\tau}$. Comparing the relative numbers of tracks and showers yields the flavor ratios $f_{\alpha, \oplus}$ [21-27]. At higher energies, flavor-specific event topologies due to $\bar{\nu}_{e}$ [24,61-64] and $\nu_{\tau}$ [65-67], already hinted at by current data $[68,69]$, might improve flavor and $\nu$ vs $\bar{\nu}$ discrimination.

Figure 2, left, shows the latest published IceCube flavor measurements [23], covering energies between $25 \mathrm{TeV}$ and $2.8 \mathrm{PeV}$, expressed via the likelihood function $\mathcal{L}_{\oplus}\left(f_{e, \oplus}, f_{\mu, \oplus}\right)$. Since precise IceCube likelihood data are not public, here and below we approximate present and future IceCube likelihood functions as twodimensional normal distributions in $f_{e, \oplus}$ and $f_{\mu, \oplus}$; unitarity demands $f_{\tau, \oplus}=1-f_{e, \oplus}-f_{\mu, \oplus}$. Because of the low statistics of present analyses, the confidence regions are broad. Because $\nu_{e^{-}}$and $\nu_{\tau^{-}}$-initiated showers are similar, they are currently not distinguished from one another on an event-by-event basis; see, however, Ref. [70]. This is why the isocontours in Fig. 2 are approximately horizontal, aligned with a direction of constant $f_{e, \oplus}+f_{\tau, \oplus}$. The degeneracy is weakly broken, because $\nu_{\tau}$ interactions create muon tracks $17 \%$ of the time, unlike $\nu_{e}$. The best fit is at $(0.49: 0.51: 0)_{\oplus}$, about $1 \sigma$ away from the nominal expectation. Later, we consider projected improvements in flavor measurement.
IceCube measures the flavor composition of the diffuse flux of high-energy astrophysical neutrinos. Since the diffuse flux is the aggregated contribution of multiple sources-which possibly emit neutrinos with different flavor ratios-the flavor ratios $f_{\alpha, \oplus}$ measured by IceCube are the average of all sources. By extension, so are the flavor ratios at the sources $f_{\alpha, S}$ that we infer below.

Inferring flavor at the sources. - For a given test choice of flavor ratios at the sources, we assess its compatibility with the data by computing an associated Bayesian posterior probability density that factors in the uncertainties in mixing parameters - via their PDFs-and the detector performance in measuring flavor ratios - via the likelihood $\mathcal{L}_{\oplus}$. The posterior probability density of $f_{\alpha, S}$ being the flavor ratios at the sources is

$$
\mathcal{P}\left(f_{\alpha, S}\right) \equiv \int d \boldsymbol{\theta} \frac{\mathcal{P}(\boldsymbol{\theta})}{\mathcal{N}(\boldsymbol{\theta})} \mathcal{L}_{\oplus}\left[f_{e, \oplus}\left(f_{\alpha, S}, \boldsymbol{\theta}\right), f_{\mu, \oplus}\left(f_{\alpha, S}, \boldsymbol{\theta}\right)\right],
$$

where $\mathcal{P}(\boldsymbol{\theta}) \equiv \mathcal{P}\left(s_{12}\right) \mathcal{P}\left(s_{23}\right) \mathcal{P}\left(s_{13}\right) \mathcal{P}\left(\delta_{C P}\right)$ are the PDFs of the mixing parameters and

$$
\begin{aligned}
& \mathcal{N}(\boldsymbol{\theta}) \\
& \equiv \int_{0}^{1} d f_{e, S} \int_{0}^{1-f_{e, S}} d f_{\mu, S} \mathcal{L}_{\oplus}\left[f_{e, \oplus}\left(f_{\alpha, S}, \boldsymbol{\theta}\right), f_{\mu, \oplus}\left(f_{\alpha, S}, \boldsymbol{\theta}\right)\right]
\end{aligned}
$$

is a normalization constant. 

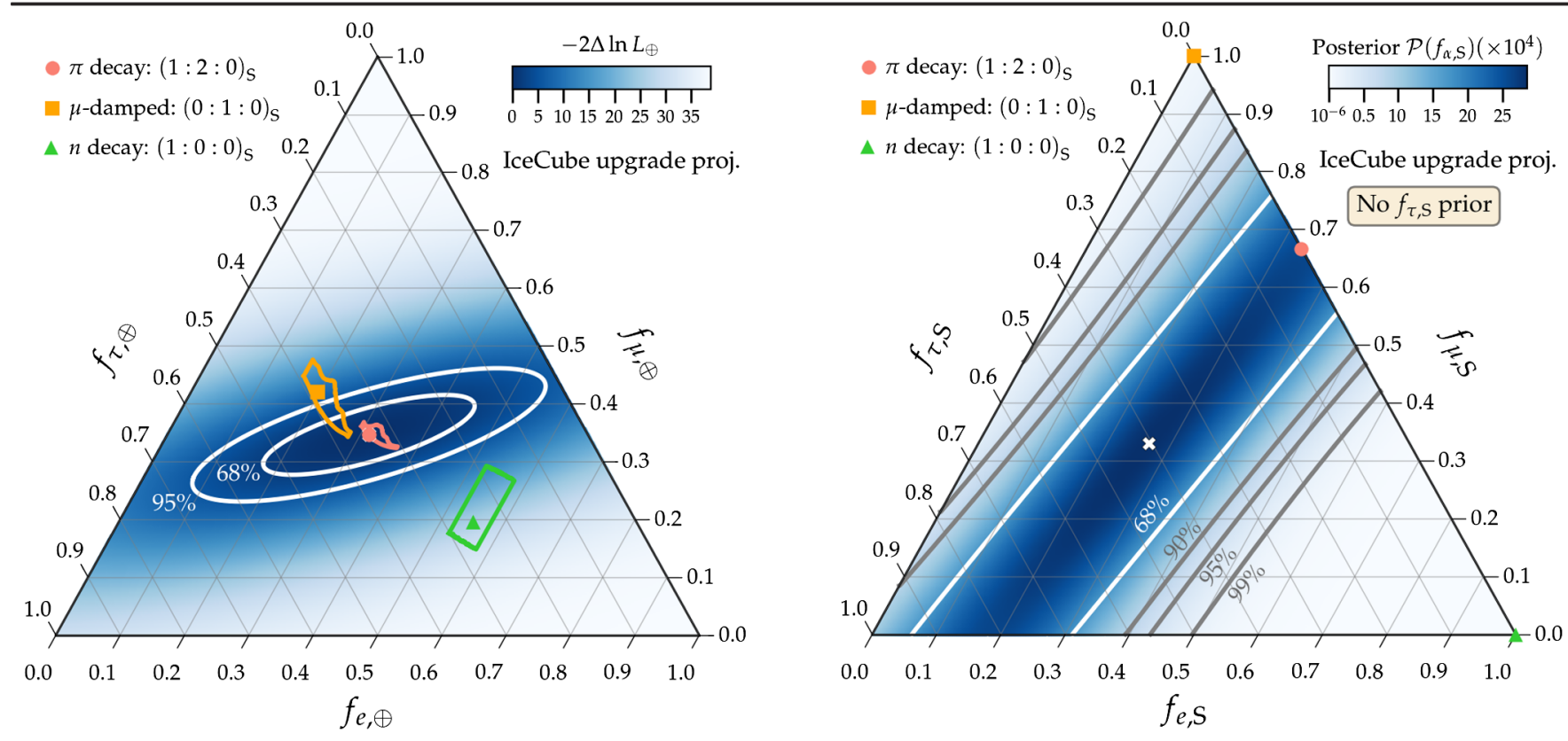

FIG. 3. Left: The same as Fig. 2, left, but showing the projected flavor sensitivity of the IceCube upgrade, approximated from Ref. [28]. Right: The same as Fig. 2, right, but showing the projected performance of the IceCube upgrade in inferring $f_{\alpha, \oplus}$.

We compute the posterior of all possible values of $f_{\alpha, S}$. After that, we calculate credible intervals of $f_{\alpha, S}$ by integrating the posterior, starting from its global maximum, down to the desired level, e.g., $68 \%, 90 \%, 95 \%$, or $99 \%$.

A previous analysis [21] also inferred the flavor composition at the sources, using early IceCube data. However, unlike the present analysis, it did not account for uncertainties in the mixing parameters, which are crucial for the interpretation of the data.

Present results.-Figure 2, right, shows the posterior of all possible flavor ratios at the sources, computed based on the latest published IceCube flavor measurements [23] (Fig. 2, left). The maximum-posterior composition is $(0: 0.7: 0.3)_{S}$, and compositions with low $f_{e, S}$ and high $f_{\mu, S}$ are preferred. This is a consequence of the current preference for low $f_{\tau, \oplus}$ in the IceCube likelihood, which maps compositions at the sources close to the $f_{e, S}=0$ axis into compositions at Earth with a high likelihood value.

Among the benchmark scenarios included in Fig. 2, production via pion decay with muon damping is allowed at the $68 \%$ credible level (Cr.L.), the full pion decay chain is slightly less favored, and neutron decay is in tension with the data, since it is allowed only at more than the 99\% Cr.L. Later, we explore how this changes if future IceCube flavor likelihood functions are centered instead on a nearly equiflavor composition.

Because the production of $\nu_{\tau}$ should be suppressed, next we supplement our method by introducing the prior $f_{\tau, S}=0$. With it, the posterior becomes a function of only $f_{e, S}$, since $f_{\mu, S}=1-f_{e, S}$. Figure 1, bottom, shows the resulting one-dimensional posterior: The maximum-posterior composition and $68 \%$ credible interval is $f_{e, S}=0.04_{-0.04}^{+0.27}$.
Future prospects.-Larger event samples, advances in flavor tagging, and reduced uncertainties in mixing parameters will significantly improve how well flavor ratios at the sources are inferred. Below, we estimate prospects for the IceCube upgrade [28] - to be built in the near future, with seven new in-fill detector strings-and for 15 years of running of the planned IceCube-Gen2 [14]—with 5-7 times the effective area.

Figure 3, left, shows the projected flavor likelihood of the IceCube upgrade [28]. Unlike the present-day likelihood, the projected one is maximum, by design, at the nominal expectation of $(0.31,0.35,0.34)_{\oplus}$, i.e., the nearly equiflavor composition at Earth expected from production by the full pion decay chain, $(1: 2: 0)_{S}$, computed using the present best-fit values of the mixing parameters. The same is true for IceCube-Gen2, though with flavor contours 5 times tighter; see Supplemental Material [44].

Figure 3, right, shows the posterior computed based on the projected likelihood of the IceCube upgrade and Fig. 3, left, without applying any prior on $f_{\tau, S}$. The maximum posterior is at $(0.25: 0.33: 0.42)_{S}$-not far from flavor equipartition-even though the IceCube likelihood was designed assuming $(1: 2: 0)_{S}$. The reason behind this is subtle but consistent with our Bayesian approach; we detail it in Supplemental Material [44]. By imposing again the prior $f_{\tau, S}=0$, we are able to sidetrack this subtlety and recover $(1: 2: 0)_{S}$ as the maximum-posterior composition.

Figure 1 shows projections for the posterior assuming $f_{\tau, S}=0$ in the IceCube upgrade and IceCube-Gen2. For IceCube-Gen2, we assume that the mixing parameters will be known with negligible uncertainties compared to the width of the likelihood. 
Assuming that neutrino production indeed occurs via pion decay, Fig. 1 shows that, in the near future, the IceCube upgrade could disfavor the muon-damped scenario at the $95 \%$ Cr.L. and the neutron-decay scenario at more than the $99 \%$ Cr.L. The uncertainty on $f_{e, S}$ is expected to shrink by a factor of 2.5. Later, in IceCube-Gen2, the uncertainty could be up to 10 times smaller than today, allowing us to single out the composition from pion decay and rule out alternatives. Supplemental Material [44] shows that, if production includes muon damping, the performance of IceCube-Gen2 will be comparable to Fig. 1. These studies could measure or constrain the average magnetic field strength in neutrino sources [71]. In reality, analyses performed at the time of operation of IceCubeGen2 will need to factor in the finite, but small, expected uncertainties in the mixing parameters.

Summary and outlook.-The study of the flavor composition of high-energy astrophysical neutrinos can help to identify their unknown production mechanism. We have introduced a method to infer the flavor composition at the neutrino sources based on measurements of the composition at Earth and on the allowed ranges of values of the neutrino mixing parameters. We hope that our results encourage neutrino-telescope collaborations, present and future, to perform further analyses in this direction.

Based on published IceCube data, we found that production of neutrinos via the decay of high-energy pions is compatible with data at the $90 \%$ credible level (Cr.L.), while the scenario where intermediate muons in the pion decay chain cool in strong magnetic fields is slightly favored, at the $68 \%$ Cr.L. Production via neutron decay is strongly disfavored, at more than $99 \%$ Cr.L.

In the future, the IceCube upgrade and extension, IceCube-Gen2, should be capable of singling out the production mechanism and firmly excluding alternatives. This will require synergy between astrophysicalneutrino experiments - to reduce uncertainties in flavor measurements-and oscillation experiments-to reduce uncertainties in neutrino mixing parameters. On both fronts, progress is ongoing, with promising prospects.

We thank Carlos Argüelles, Siqiao $\mathrm{Mu}$, and Sergio Palomares-Ruiz for useful discussion. M. A. and M. B. are supported by the Danmarks Grundforskningsfond Grant No. 1041811001 and Villum Fonden (Projects No. 18994 and No. 13164, respectively). This work used resources provided by the High Performance Computing Center at the University of Copenhagen.

*mbustamante@nbi.ku.dk ${ }^{\dagger}$ markus.ahlers@nbi.ku.dk

[1] L. A. Anchordoqui et al., Cosmic neutrino Pevatrons: A brand new pathway to astronomy, astrophysics, and particle physics, J. High Energy Astrophys. 1-2, 1 (2018).
[2] M. Ahlers and F. Halzen, Opening a new window onto the universe with IceCube, Prog. Part. Nucl. Phys. 102, 73 (2018).

[3] M. G. Aartsen et al. (IceCube Collaboration), First Observation of PeV-Energy Neutrinos with IceCube, Phys. Rev. Lett. 111, 021103 (2013).

[4] M. G. Aartsen et al. (IceCube Collaboration), Evidence for high-energy extraterrestrial neutrinos at the IceCube detector, Science 342, 1242856 (2013).

[5] M. G. Aartsen et al. (IceCube Collaboration), Observation of High-Energy Astrophysical Neutrinos in Three Years of IceCube Data, Phys. Rev. Lett. 113, 101101 (2014).

[6] M. G. Aartsen et al. (IceCube Collaboration), Evidence for Astrophysical Muon Neutrinos from the Northern Sky with IceCube, Phys. Rev. Lett. 115, 081102 (2015).

[7] M. G. Aartsen et al. (IceCube Collaboration), Observation and characterization of a cosmic muon neutrino flux from the northern hemisphere using six years of IceCube data, Astrophys. J. 833, 3 (2016).

[8] M. Ahlers and K. Murase, Probing the galactic origin of the IceCube excess with gamma-rays, Phys. Rev. D 90, 023010 (2014).

[9] M. Ahlers, Y. Bai, V. Barger, and R. Lu, Galactic neutrinos in the $\mathrm{TeV}$ to $\mathrm{PeV}$ range, Phys. Rev. D 93, 013009 (2016).

[10] P. B. Denton, D. Marfatia, and T. J. Weiler, The galactic contribution to IceCube's astrophysical neutrino flux, J. Cosmol. Astropart. Phys. 08 (2017) 033.

[11] M. G. Aartsen et al. (IceCube Collaboration), Constraints on Galactic neutrino emission with seven years of IceCube data, Astrophys. J. 849, 67 (2017).

[12] M. G. Aartsen et al. (Liverpool Telescope, MAGIC, H.E.S.S., AGILE, Kiso, VLA/17B-403, INTEGRAL, Kapteyn, Subaru, HAWC, Fermi-LAT, ASAS-SN, VERITAS, Kanata, IceCube, and Swift NuSTAR Collaborations), Multimessenger observations of a flaring blazar coincident with high-energy neutrino IceCube-170922A, Science 361, eaat1378 (2018).

[13] M. G. Aartsen et al. (IceCube Collaboration), Neutrino emission from the direction of the blazar TXS $0506+$ 056 prior to the IceCube-170922A alert, Science 361, 147 (2018).

[14] M. G. Aartsen et al. (IceCube Collaboration), IceCubeGen2: A vision for the future of neutrino astronomy in Antarctica, arXiv:1412.5106.

[15] S. Adrian-Martinez et al. (KM3Net Collaboration), Letter of intent for KM3NeT 2.0, J. Phys. G 43, 084001 (2016).

[16] G. Barenboim and C. Quigg, Neutrino observatories can characterize cosmic sources and neutrino properties, Phys. Rev. D 67, 073024 (2003).

[17] Z.-Z. Xing and S. Zhou, Towards determination of the initial flavor composition of ultrahigh-energy neutrino fluxes with neutrino telescopes, Phys. Rev. D 74, 013010 (2006).

[18] S. Pakvasa, W. Rodejohann, and T. J. Weiler, Flavor ratios of astrophysical neutrinos: Implications for precision measurements, J. High Energy Phys. 02 (2008) 005.

[19] K.-C. Lai, G.-L. Lin, and T. C. Liu, Determination of the neutrino flavor ratio at the astrophysical source, Phys. Rev. D 80, 103005 (2009). 
[20] S. Choubey and W. Rodejohann, Flavor composition of UHE neutrinos at source and at neutrino telescopes, Phys. Rev. D 80, 113006 (2009).

[21] O. Mena, S. Palomares-Ruiz, and A.C. Vincent, Flavor Composition of the High-Energy Neutrino Events in IceCube, Phys. Rev. Lett. 113, 091103 (2014).

[22] M. G. Aartsen et al. (IceCube Collaboration), Flavor Ratio of Astrophysical Neutrinos above $35 \mathrm{TeV}$ in IceCube, Phys. Rev. Lett. 114, 171102 (2015).

[23] M. G. Aartsen et al. (IceCube Collaboration), A combined maximum-likelihood analysis of the high-energy astrophysical neutrino flux measured with IceCube, Astrophys. J. 809, 98 (2015).

[24] A. Palladino and F. Vissani, The natural parameterization of cosmic neutrino oscillations, Eur. Phys. J. C 75, 433 (2015).

[25] S. Palomares-Ruiz, A. C. Vincent, and O. Mena, Spectral analysis of the high-energy IceCube neutrinos, Phys. Rev. D 91, 103008 (2015).

[26] A. C. Vincent, S. Palomares-Ruiz, and O. Mena, Analysis of the 4-year IceCube high-energy starting events, Phys. Rev. D 94, 023009 (2016).

[27] M. G. Aartsen et al. (IceCube Collaboration), Measurements using the inelasticity distribution of multi-TeV neutrino interactions in IceCube, Phys. Rev. D 99, 032004 (2019).

[28] Summer Blot, IceCube upgrade and Gen2, in Proceedings of TeVPA 2018, Berlin, Germany (unpublished), http:// tevpa2018.desy.de.

[29] M. Kowalski, Next-generation IceCube, in Proceedings of the Neutrino Oscillation Workshop (NOW) 2018, Ostuni, Italy (unpublished), http://www.ba.infn.it/ now/.

[30] S. H. Margolis, D. N. Schramm, and R. Silberberg, Ultrahighenergy neutrino astronomy, Astrophys. J. 221, 990 (1978).

[31] F. W. Stecker, Diffuse fluxes of cosmic high-energy neutrinos, Astrophys. J. 228, 919 (1979).

[32] S. R. Kelner, F. A. Aharonian, and V. V. Bugayov, Energy spectra of gamma-rays, electrons and neutrinos produced at proton-proton interactions in the very high energy regime, Phys. Rev. D 74, 034018 (2006); Erratum, Phys. Rev. D 79, 039901(E) (2009).

[33] A. Mücke, R. Engel, J. P. Rachen, R. J. Protheroe, and T. Stanev, SOPHIA: Monte Carlo simulations of photohadronic processes in astrophysics, Comput. Phys. Commun. 124, 290 (2000).

[34] S. Hümmer, M. Rüger, F. Spanier, and W. Winter, Simplified models for photohadronic interactions in cosmic accelerators, Astrophys. J. 721, 630 (2010).

[35] O. Mena, I. Mocioiu, and S. Razzaque, Oscillation effects on high-energy neutrino fluxes from astrophysical hidden sources, Phys. Rev. D 75, 063003 (2007).

[36] S. Razzaque and A. Yu. Smirnov, Flavor conversion of cosmic neutrinos from hidden jets, J. High Energy Phys. 03 (2010) 031.

[37] S. Sahu and B. Zhang, Effect of resonant neutrino oscillation on $\mathrm{TeV}$ neutrino flavor ratio from choked GRBs, Res. Astron. Astrophys. 10, 943 (2010).

[38] K. Varela, S. Sahu, A. F. O. Oliveros, and J. C. Sanabria, High energy neutrinos from choked GRBs and their flavor ratio measurement by the IceCube, Eur. Phys. J. C 75, 289 (2015).
[39] D. Xiao and Z. G. Dai, TeV-PeV neutrino oscillation of lowluminosity gamma-ray bursts, Astrophys. J. 805, 137 (2015).

[40] T. Kashti and E. Waxman, Flavoring Astrophysical Neutrinos: Flavor Ratios Depend on Energy, Phys. Rev. Lett. 95, 181101 (2005).

[41] P. Lipari, M. Lusignoli, and D. Meloni, Flavor composition and energy spectrum of astrophysical neutrinos, Phys. Rev. D 75, 123005 (2007).

[42] S. Hümmer, P. Baerwald, and W. Winter, Neutrino Emission from Gamma-Ray Burst Fireballs, Revised, Phys. Rev. Lett. 108, 231101 (2012).

[43] M. Bustamante, J. F. Beacom, and W. Winter, Theoretically Palatable Flavor Combinations of Astrophysical Neutrinos, Phys. Rev. Lett. 115, 161302 (2015).

[44] See Supplemental Material at http://link.aps.org/ supplemental/10.1103/PhysRevLett.122.241101 for details about the projected posterior probability density, IceCubeGen2 projections, and results obtained assuming the inverted mass neutrino ordering.

[45] M. G. Aartsen et al. (IceCube Collaboration), Energy reconstruction methods in the IceCube neutrino telescope, J. Instrum. 9, P03009 (2014).

[46] S. Pakvasa, Neutrino flavor goniometry by high energy astrophysical beams, Mod. Phys. Lett. A 23, 1313 (2008).

[47] A. Esmaili and Y. Farzan, An analysis of cosmic neutrinos: Flavor composition at source and neutrino mixing parameters, Nucl. Phys. B821, 197 (2009).

[48] M. Bustamante, A. M. Gago, and C. P. Garay, Energyindependent new physics in the flavour ratios of highenergy astrophysical neutrinos, J. High Energy Phys. 04 (2010) 066.

[49] X.-J. Xu, H.-J. He, and W. Rodejohann, Constraining astrophysical neutrino flavor composition from leptonic unitarity, J. Cosmol. Astropart. Phys. 12 (2014) 039.

[50] C. A. Arguelles, T. Katori, and J. Salvadó, New Physics in Astrophysical Neutrino Flavor, Phys. Rev. Lett. 115, 161303 (2015).

[51] I. M. Shoemaker and K. Murase, Probing BSM neutrino physics with flavor and spectral distortions: Prospects for future high-energy neutrino telescopes, Phys. Rev. D 93, 085004 (2016).

[52] M. Bustamante, J. F. Beacom, and K. Murase, Testing decay of astrophysical neutrinos with incomplete information, Phys. Rev. D 95, 063013 (2017).

[53] V. Brdar, J. Kopp, and X.-P. Wang, Sterile neutrinos and flavor ratios in IceCube, J. Cosmol. Astropart. Phys. 01 (2017) 026.

[54] P. F. de Salas, R. A. Lineros, and M. Tórtola, Neutrino propagation in the Galactic dark matter halo, Phys. Rev. D 94, 123001 (2016).

[55] R. W. Rasmussen, L. Lechner, M. Ackermann, M. Kowalski, and W. Winter, Astrophysical neutrinos flavored with beyond the Standard Model physics, Phys. Rev. D 96, 083018 (2017).

[56] M. Bustamante and S. K. Agarwalla, Universe's Worth of Electrons to Probe Long-Range Interactions of High-Energy Astrophysical Neutrinos, Phys. Rev. Lett. 122, 061103 (2019). 
[57] M. Ahlers, M. Bustamante, and S. Mu, Unitarity bounds of astrophysical neutrinos, Phys. Rev. D 98, 123023 (2018).

[58] I. Esteban, M. C. González-García, M. Maltoni, I. MartínezSoler, and T. Schwetz, Updated fit to three neutrino mixing: Exploring the accelerator-reactor complementarity, J. High Energy Phys. 01 (2017) 087.

[59] NuFit, Three-neutrino fit based on data available in January 2018, http://www.nu-fit.org/.

[60] M. G. Aartsen et al. (IceCube Collaboration), The IceCube neutrino observatory: Instrumentation and online systems, J. Instrum. 12, P03012 (2017).

[61] S. L. Glashow, Resonant Scattering of Antineutrinos, Phys. Rev. 118, 316 (1960).

[62] L. A. Anchordoqui, H. Goldberg, F. Halzen, and T. J. Weiler, Neutrinos as a diagnostic of high energy astrophysical processes, Phys. Lett. B 621, 18 (2005).

[63] A. Bhattacharya, R. Gandhi, W. Rodejohann, and A. Watanabe, The Glashow resonance at IceCube: Signatures, event rates and $p p$ vs. $p \gamma$ interactions, J. Cosmol. Astropart. Phys. 10 (2011) 017.

[64] V. Barger, L. Fu, J. G. Learned, D. Marfatia, S. Pakvasa, and T. J. Weiler, Glashow resonance as a window into cosmic neutrino sources, Phys. Rev. D 90, 121301(R) (2014).
[65] J. G. Learned and S. Pakvasa, Detecting $\nu_{\tau}$ oscillations at PeV energies, Astropart. Phys. 3, 267 (1995).

[66] J. F. Beacom, N. F. Bell, D. Hooper, S. Pakvasa, and T. J. Weiler, Measuring flavor ratios of high-energy astrophysical neutrinos, Phys. Rev. D 68, 093005 (2003); Erratum, Phys. Rev. D 72, 019901(E) (2005).

[67] E. Bugaev, T. Montaruli, Y. Shlepin, and I. A. Sokalski, Propagation of $\tau$-neutrinos and $\tau$-leptons through the earth and their detection in underwater/ice neutrino telescopes, Astropart. Phys. 21, 491 (2004).

[68] J. Stachurska, New Measurement of the flavor composition of high-energy neutrino events with contained vertices in IceCube, in Proceedings of TeVPA 2018, Berlin (unpublished), http://tevpa2018.desy.de.

[69] L. Lu, Recent IceCube results-Evidences of neutrino emission from the blazar TXS $0506+056$ and searches for Glashow resonance, in Proceedings of UHECR 2018, Paris (unpublished), https://indico.in2p3.fr/event/17063/.

[70] S. W. Li, M. Bustamante, and J. F. Beacom, Echo Technique to Distinguish Flavors of Astrophysical Neutrinos, Phys. Rev. Lett. 122, 151101 (2019).

[71] P. Baerwald, S. Hümmer, and W. Winter, Systematics in the interpretation of aggregated neutrino flux limits and flavor ratios from gamma-ray bursts, Astropart. Phys. 35, 508 (2012). 